\title{
CURVELET DECOMPOSITION FOR DETECTION OF CYLINDRICAL TARGETS
}

\author{
Frank Crosby \\ Naval Surface Warfare Center - Panama City, Panama City Florida
}

\begin{abstract}
Curvelets are a multiscale system with very high directional sensitivity. A new detection algorithm is herein described which operates on a curvelet decomposition of acoustic imagery. The algorithm detects the presence of cylindrical targets through a statistical mapping of curvelet coefficients. The coefficients are calculated as an inner product between image features and a curvelet basis element. The similarity in appearance between cylindrical targets and curvelet basis elements yield an accurate detection algorithm with a very low false alarm rate.
\end{abstract}

Index Terms- Acoustic applications, Wavelet transforms, Underwater object detection

\section{INTRODUCTION}

The curvelet transform has only been developed in the last few years and is an attempt to overcome inherent limitations of traditional multiscale representations such as wavelets. Curvelets are an extension of wavelets that include the ability to characterize image areas.

Conceptually, the curvelet transform is a multiscale pyramid with many directions and positions at each length scale, and needle-shaped elements at fine scales. This pyramid is nonstandard, however. Curvelets have useful geometric features that set them apart from wavelets. Curvelets overcome limited directionality through the use basis elements that exhibit very high directional sensitivity. Curvelets are reviewed in Section 2.

This paper presents a new detection method using a curvelet decomposition and statistical estimation to perform target detection of cylindrical targets where high resolution features are available. Cylindrical targets present unique detection difficulties since they have variable sizes and their signatures are orientation dependent.

High-resolution acoustic sensors are being investigated to improve sea mine detection and classification. Synthetic aperture sonar (SAS) has emerged as a leading candidate in revealing fine details of underwater objects. The computer aided detection and computer aided classification
(CAD/CAC) employed by lower-resolution sensors rely on coarse features [1].

Synthetic aperture sonar achieves high resolution in the cross range dimension by taking advantage of the motion of the vehicle carrying the sonar to synthesize the effect of a large receiver aperture. The range resolution is a function of the bandwidth [2].

Almost all sonar detection systems depend on operator involvement. The goal of $\mathrm{CAD} / \mathrm{CAC}$ has traditionally been to alert operators to suspicious objects. In a real-time bandwidth limited system imagery of suspicious objects may be transmitted to an operator. However, only transmitting a clip removes context, which can limited operator interpretation. For example, a single sand ripple may look like a cylindrical target without its surroundings.

This detection algorithm employs a different paradigm. Rather than identifying suspicious objects, suspicious images are identified. The goal is to reduce the number of images, not necessarily the number of objects, that an operator must review. Reduced imagery is achieved through a very low and reliable false alarm rate. Furthermore, robustness is achieved by having minimal subjective parameters.

The detection algorithm finds cylindrical targets with unknown orientation and some size variability. Each sonar image is decomposed using a curvelet basis. The decomposition gives a set of sub-images for a series of rotations and scales. Each subimage is mapped to a common interval for comparison. The algorithm, presented in Section 3, searches for the best curvelet basis function match among the sub-images. Our algorithm is compared to ground truth in Section 4. The results cover two large and environmental distinct data sets.

\section{CURVELETS}

Wavelets have been successful in many areas of image processing when the image data is generally in hierarchical structure. Using multiscale approaches such as wavelets, an image can be decomposed into components at different scales. The curvelet transform is a multiscale pyramid with many directions and positions at each length scale.

Curvelets are defined in dimensions greater than 1. A starting point for consideration is two-dimensional wavelets. A popular 
and simple method of constructing a two-dimensional basis on $L^{2}\left(\mathfrak{R}^{2}\right)$ is to form the tensor product of 1-dimensional scaling functions $\varphi(x), x \in \mathfrak{R}$.

Bandlimited wavelets are one family of possible functions. The corresponding two-dimensional scaling function is given by a square in the Fourier domain defined by $\hat{\Phi}(x, y)=\hat{\varphi}(x) \hat{\varphi}(y)$. Curvelets were originally defined using cross-sections of the square support defined by $\hat{\Phi}$. The cross-sections were calculated using ridgelets [3]. Construction of curvelets using ridgelets involves a complicated indexing structure which can hinder their appreciation.

The most recent curvelet constructions are based on a polar coordinate system in the frequency domain. Radial and angular windows are formed to provide orthogonal compactly supported windows using scales, rotations, and translations in much the same way that bandlimited wavelets form a basis using only scales and translations. The polar coordinate tiling is shown in Figure 1.

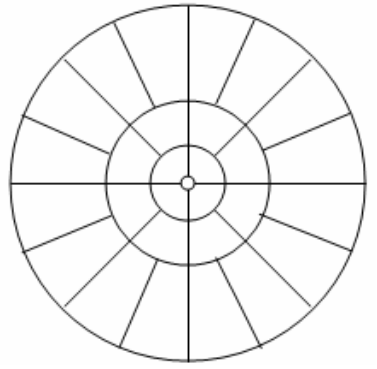

Figure 1: Frequency space tiling

The radial window, $W(\mathrm{r})$, and an angular window $V(\mathrm{t})$ obey the admissibility conditions:

$$
\begin{gathered}
\sum_{j=-\infty}^{j=\infty} W^{2}\left(2^{j} r\right)=1, \quad r>0 \\
\sum_{j=-\infty}^{j=\infty} V^{2}(t-2 \pi l)=1, \quad t \in \mathfrak{R}
\end{gathered}
$$

As in wavelet theory, we introduce the lowpass window $W_{j_{0}}$ which satisfies the following condition: $W_{j_{0}}(r)^{2}+\sum_{j>j_{0}} W\left(2^{-j} r\right)^{2}=1$.

The Meyer wavelets are one family of possible functions. They are defined in the frequency domain and are smoothed versions of bandlimted wavelets.

For each $j \geq j_{0}, W\left(2^{-j} r\right)$ smoothly extracts the frequency content inside the dyadic region $\left(2^{j-1}, 2^{j+1}\right)$.
Curvelets are organized by the triple index $(j, l, k)$, with $\mathrm{j}$ standing for scale, 1 for orientation and $k=\left(k_{1}, k_{2}\right)$ for translation.

The coarsest scale curvelet, $j=j_{0}$ is isotropic and the only index for $l$ is zero. We define the frequency radial window

$$
U_{j_{0}}(r, \theta)=W_{j_{0}}(r, \theta)
$$

A Fourier basis for each of the wedges defined by $U_{j_{0}}(r, \theta)$ is found by multiplying the wedge by a local Fourier basis determined by an enclosing rectangle. Suppose $U_{j_{0}, 0}$ is supported on a rectangle of size $L_{1, j_{0}} \times L_{2, j_{0}}$. Using the basis

$$
\begin{aligned}
& \exp \left[2 \pi i\left(k_{1}\left(r e^{i \theta}\right)_{1} / L_{1, j_{0}}\right]\right. \\
& \times \exp \left[2 \pi i\left(k_{2}\left(r e^{i \theta}\right)_{2} / L_{2, j_{0}}\right]\right.
\end{aligned}
$$

the coarsest curvelets are defined by means of their Fourier Transform

$$
\begin{aligned}
& \hat{\varphi}_{j_{0}, 0, k}(r, \theta)=U_{j_{0}, 0}(r, \theta) \\
& \cdot \exp \left[-2 \pi i\left(k_{1}\left(r e^{i \theta}\right)_{1} / L_{1, j_{0}}+k_{2}\left(r e^{i \theta}\right)_{2} / L_{2, j_{0}}\right)\right] \\
& / \sqrt{L_{1, j_{0}} \cdot L_{2, j_{0}}} .
\end{aligned}
$$

For finer scales, defined as $j>j_{0}$, the frequency content radially extracted by $W\left(2^{-j} r\right)$ is further partitioned into $2^{j / 2}$ angular windows using $V$. For each $l$ : $0 \leq l \leq 2^{j / 2}$, a wedge like frequency window $U_{j, l}(r, \theta)$ is defined by

$$
U_{j}(r, \theta)=W\left(2^{-j} r\right) V\left(2^{j / 2}\left(\theta-\theta_{l}\right)\right)
$$

Where $\theta_{l}=2 \pi l \cdot 2^{-j / 2}$.

Again multiplying a local Fourier basis and the window, we suppose that $U_{j, l}(r, \theta)$ is supported on a rectangle of size $L_{1, j}$ by $L_{2, j}$.

The curvelets at scale $\mathrm{j}$ and orientation $l=0$ are defined in frequency space as

$$
\begin{aligned}
& \hat{\varphi}_{j, 0, k}(r, \theta)=U_{j, 0}(r, \theta) \\
& \cdot \exp \left[-2 \pi i\left(k_{1}\left(r e^{i \theta}\right)_{1} / L_{1, j_{0}}+k_{2}\left(r e^{i \theta}\right)_{2} / L_{2, j_{0}}\right)\right] \\
& / \sqrt{L_{1, j} \cdot L_{2, j}}
\end{aligned}
$$

From this definition, for any $k$, with $k \in \mathbf{Z}^{2}$, $\varphi_{j, 0, k}=\varphi_{j, 0,0}\left(x-\left(k_{1} / L_{1, j}, k_{2} / L_{2, j}\right)\right)$

For a general $l$, the curvelets of orientation $l$ are defined by $\varphi_{j, l, k}=\varphi_{j, 0, k}\left(R_{-\theta_{l}} \cdot x\right)$ where $R_{\theta}$ is the rotation through $\theta$ radians. 
The curvelet coefficients of a function $f \in L^{2}\left(\Re^{2}\right)$ are the inner products

$$
c(j, l, k) \equiv\left\langle f, \varphi_{j, l, k}\right\rangle=\int f(x) \overline{\varphi_{j, l, k}(x)} d x .
$$

The coarsest scale curvelets are non-directional. However, it is the behavior of the fine-scale directional elements that are of interest.

Curvelets are real-valued. The envelope is concentrated near a vertical ridge of length about $2^{-j}$ and width $2^{-2 j}$.

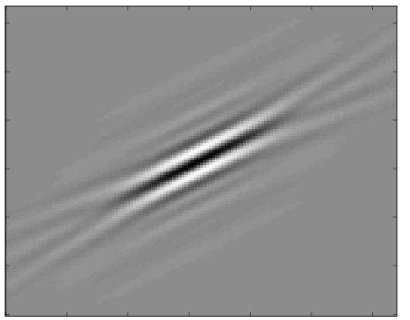

Figure 2: Inverse of a Curvelet Basis Element.

\section{CURVELET SPACE TARGET DETECTION}

Curvelets are nearly ideally suited for detecting cylindrical targets. Their basis elements closely match the appearance of such targets. Cylindrical targets are visible in sonar imagery as reflective linear elements. Figure 3 is an example of this type of target. The scale and directional variability present in imagery is inherent in curvelet construction.

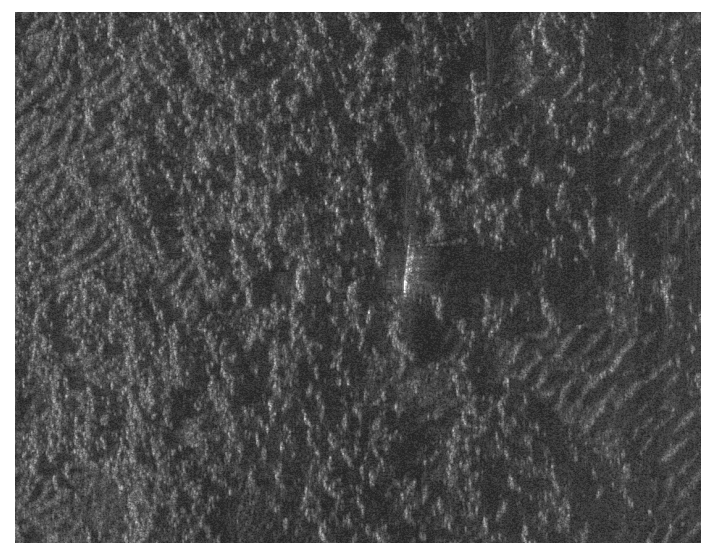

Figure 3: SAS cylindrical target image.

The expected target size is bounded by a scale interval which determines are range of sub-images to be searched. Directionality is more variable and so, many directions are searched at each scale.

A curvelet decomposition of an image consists of a set of scale and rotation sub-images. Each image represents the set of translations of a scale-rotation basis element. The curvelet implementation used to produce our results is CurveLab [4].

Target detection is achieved by a search of each sub-image within an expected target size interval. Each sub-image is statistically modeled to determine the presence of anomalous pixels.

Curvelets coefficients can vary greatly across rotations and scales. The curvelet sub-images produced by CurveLab are different sizes. In order to compare the various sub-images and select the only that best captures a target, if it exists; we need a mapping to a common reference set. The mapping is done using a statistical model whose parameters are estimated for each subimage.

\subsection{Statistical Model}

The mapping is selected based on the exponential distribution. The probability density function corresponding to the exponential distribution is $g(x)=\frac{1}{\mu} e^{-x / \mu}$. The parameter $\mu$ is the image mean. As such, the distribution shifts independently from image to image. The mean is estimated using the detection values as discreet samples of the continuous exponential distribution. A histogram from a curvelet sub-image is shown in Figure 4. The histogram is fit with an exponential distribution. Through out the data presented in Section 4, similar high quality fits were obtained. Larger pixel values in the sub-image represent closer matches between image features and curvelet basis elements. In general, most of the image does not match particular basis elements.

The predicted number of abnormal pixels per image is calculated using the distribution function, $G$, and the number of pixels in the sub-image, M. The distribution function is given by

$$
G(x)=\left\{\begin{array}{cc}
0, & -\infty<x<0, \\
1-e^{-x / \mu} & 0 \leq x<\infty .
\end{array}\right.
$$

The number of elements that are expected to be greater than a threshold, $\mathrm{T}$, is given by $(1-G(\mathrm{~T})) M$.

If $K$ is the desired number of false alarms then $\mathrm{T}$ is found by

$$
\begin{gathered}
(1-G(\mathrm{~T})) N<K \\
\mathrm{~T} \geq-\mu \ln (K / N)
\end{gathered}
$$

The threshold is independent of the scale of the curvelet values and sub-image size. All sub-images can be compared relative to a single threshold.

\subsection{Best Match Search}

First, a threshold is selected for all processing. This threshold represents the only algorithm parameter. Each sonar image is decomposed. The decomposition is searched for the one subimage with the best curvelet basis element match realized as 
pixels above the threshold. A further screening groups neighboring pixels to generate a single detection.

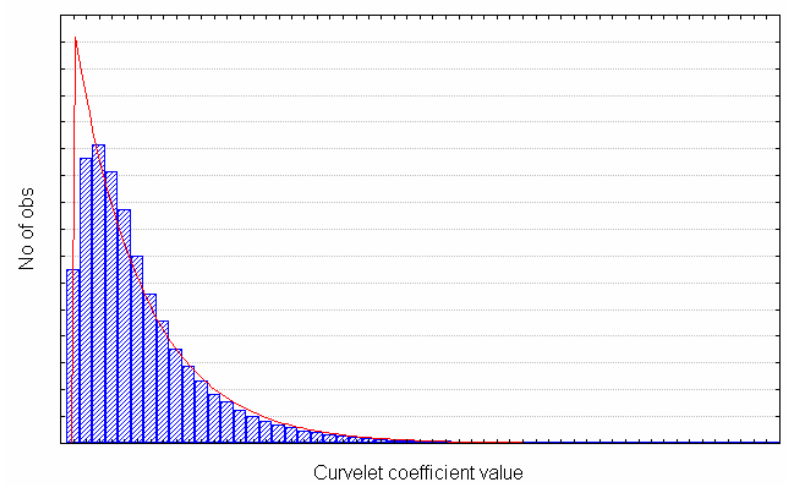

Figure 4: Curvelet sub-image Histogram with exponential distribution fit.

It is possible that two objects with different orientations are contained in a single image. With our search, the stronger detection will mask the other object. However, this is not inconsistent as the image is deemed suspicious and subject to further processing or inspection.

\section{RESULTS}

The cylindrical target detection algorithm has been tested on two different data sets. One was collected in the Gulf of Mexico and the other in the Mediterranean near La Spezia and Levanto, Italy. The background for both image sets is benign sand. The Gulf of Mexico data set has 399 images with cylindrical targets in 11 of them. The Mediterranean data set has 220 images with cylindrical targets in 6 images.

The algorithm labels images as possessing targets or being clear. Both data sets had a variety of targets. Although the detection algorithm is designed for cylindrical targets, other targets can appear cylindrical at certain orientations. When images without cylindrical targets were detected by the algorithm they were judged as false alarms.

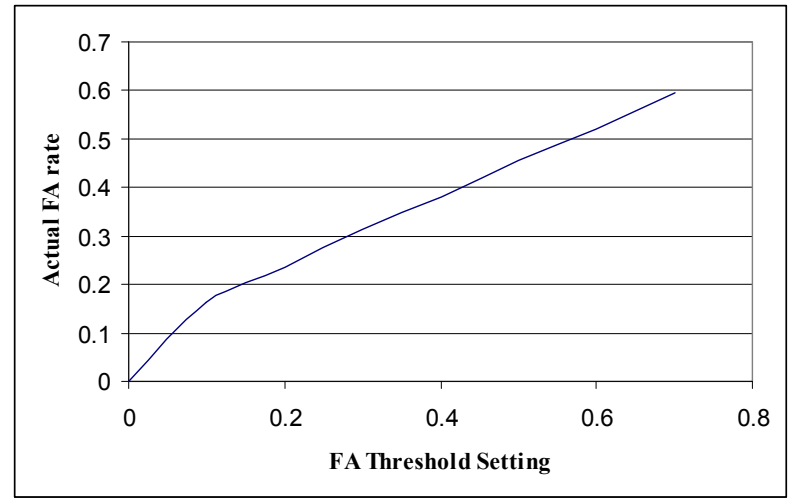

Figure 5: Actual FA rate versus threshold setting.

The graph in Figure 5 shows the combined false alarm for both sets versus the threshold settings. The graph is close to a linear relationship. But, the grouping of neighboring pixels reduces the false alarm rate. The graph in Figure 6 is a receiver operating characteristic chart that shows detection percentage versus the false alarm (FA) rate.

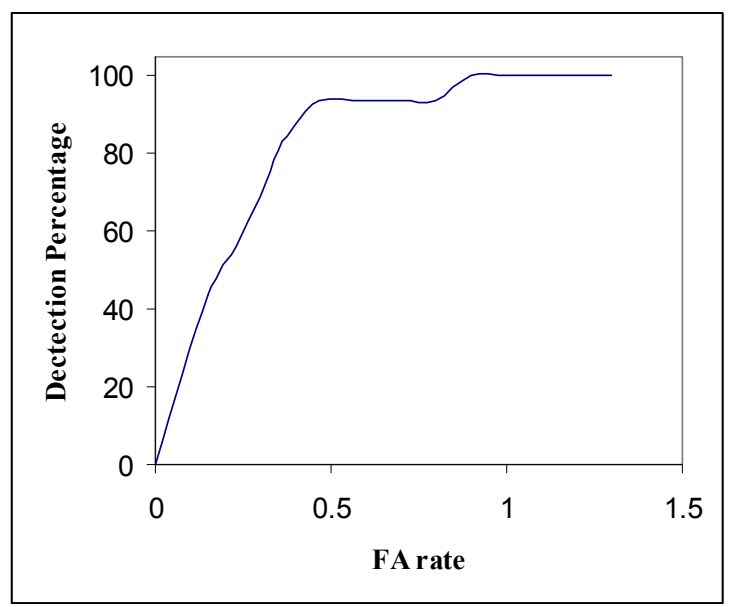

Figure 6: Receiver Operating Characteristic

\section{CONCLUSION}

The curvelet space detection algorithm that we have presented identifies images that contain cylindrical targets. It seeks to identify the best match per image. If there are no matches, we found that the image feature was not present. If the feature was present, secondary features are not sought. A follow-on algorithm would be necessary to further interrogate the images for all possible targets.

The curvelet space detection algorithm that we have presented has a very low false alarm rate, while maintaining nearly perfect detection.

\section{REFERENCES}

[1] G. J. Dobeck, "Algorithm Fusion for the Detection and Classification of Sea Mines in the Very Shallow Water Region using Side-Scan Sonar Imagery", Proceedings of SPIE, vol. 4038, pp. 438-361, April, 2000.

[2] P. T. Gough and D. W. Hawkins, "A Short History of Synthetic Aperture Sonar", International Geoscience and Remote Sensing Symposium (IGARSS) vol. 2 pp 618-620, 1998.

[3] E. J. Candès and D. L. Donoho, "Curvelets - a Surprisingly Effective Nonadaptive Representation for Objects with Edges", Curves and Surfaces, Vanderbilt University Press, 2000, Nashville, TN.

[4] Emmanuel Candès, Laurent Demanet, David Donoho, Lexing Ying, CurveLab http://www.curvelet.org 